\title{
VLP-encapsulated TLR9 Agonist CMP-001
}

National Cancer Institute

\section{Source}

National Cancer Institute. VLP-encapsulated TLR9 Agonist CMP-001. NCI Thesaurus.

Code C126422.

An agent composed of an unmethylated CpG motif-rich $\mathrm{G} 10$ olig onucleotide, which is an agonist of toll-like receptor 9 (TLR9), encapsulated in noninfectious virus-like particles (VLPs), with potential immunostimulating and antineoplastic activities. Upon administration of CMP-001, the VLPs are specifically taken up by and release the oligonucleotide into antigen-presenting cells (APCs), including dendritic cells (DCs). In turn, the olig onucleotide binds to and activates intracellular TLR9. This stimulates immune signaling pathways, induces the innate immune system and may promote the immune system to attack tumor cells. VLPs stimulate the immune system. TLR9, a member of the TLR family, plays a key role in both pathogen recognition and the activation of innate immunity. 